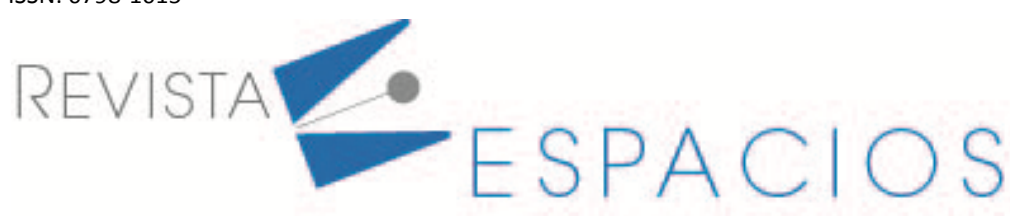

Vol. 42 (08) $2021 \cdot$ Art. 2

\title{
Dimensiones del clima laboral que afectan la ejecución de proyectos. Caso BID (Ecuador)
}

\section{Dimensions of the work environment that affect the execution of projects. IDB case}

\author{
VILLAFUERTE, Santiago B. ${ }^{1}$ \\ VITERI, Fernando A. ${ }^{2}$ \\ LOPEZ, Héctor $A .^{3}$
}

\begin{abstract}
Resumen
La ejecución de los proyectos BID, en coordinación con sus distintos actores, presentan dificultades atribuidas según la Gerencia a fallas de comunicación, excesiva estructura burocrática, desconocimiento en la asignación, delimitación de funciones, responsabilidades de sus integrantes, desvinculaciones en el sector público, incumplimiento de normas, procesos, políticas, así como, movimientos internos de personal que provocan alteraciones en las fases preparatorias, precontractual, contractual y postcontractual; siendo el clima laboral, según Robles, Dierssen, Martínez, Herrera, Díaz, y Llorca (2005) un conjunto de características que influyen en el comportamiento de una institución y en la realización de sus procesos y proyectos que la distingue de otras; siendo éstas de permanencia relativa en el tiempo. Robbins (2009) señaló que el clima laboral genera satisfacción en el colaborador y en igual sentido fuera de él, entendiendo que una meta de alta satisfacción puede expresarse en términos, tanto de dinero, como de responsabilidad social, por tanto, si cualquier persona del equipo de gestión está motivado aportará al logro de los objetivos del Proyecto creando de esta manera una coordinación de logro de objetivos institucionales y objetivos personales.
\end{abstract}

Palabras clave: ambiente laboral, clima laboral, proyecto, comportamiento.

\section{Abstract}

The research was designed with the objective of analyzing the work environment (climate) and its impact on the execution of projects of the Inter-American Development Bank (IDB). The questionnaire was used as a data collection tool and the correlational analysis identified elements to strengthen in the work environment in the execution of IDB projects, with the adaptation of officials, well-being and working conditions being the fundamental components to improve behavior and management team productivity.

key words: work environment, work environment, project, behavior.

\section{Introducción}

La ejecución de los proyectos BID en coordinación con sus distintos actores presentan dificultades atribuidas según la Gerencia a fallas de comunicación, excesiva estructura burocrática, desconocimiento en la asignación, delimitación de funciones, responsabilidades de sus integrantes, desvinculaciones en el sector público, incumplimiento de normas, procesos, políticas, así como, movimientos internos de personal que provocan

\footnotetext{
${ }^{1}$ Analista de Proyectos, Ministerio de Salud Publica; Magíster en Administración de Empresas de la Universidad Internacional del Ecuador; savillafuertepa@uide.edu.ec; 0000-0002-7996-9900

2 Jefe de Taller, RV Servivios Automotrices; Magíster en Administración de Empresas de la Universidad Internacional del Ecuador; feviterivi@uide.edu.ec; ORCID: 0000-0001-8619-0906

${ }^{3}$ Docente de Posgrados, Universidad Internacional del Ecuador; Doctorando en Dirección de Empresas, Universidad de Valencia; helopezpa@uide.edu.ec; ORCID: 0000-0003-0456-5271
} 
alteraciones en las fases preparatorias, precontractual, contractual y postcontractual; siendo el clima laboral según Robles, Dierssen, Martínez, Herrera, Díaz, y Llorca (2005) un grupo de características que influyen en el compratmiento de una institución, la realización de sus procesos y proyectos que la distingue de otras; siendo de permanencia relativa en el tiempo. Robbins (2009) señaló que el clima laboral genera satisfacción en el colaborador y en igual sentido fuera de el, entendiendo que una meta de una alta satisfacción puede expresarse en términos tanto de dinero como de responsabilidad social, por tanto, si cualquier persona del equipo de gestión está motivado, aportará al logro de los objetivos del Proyecto creando de esta manera una coordinación de logro de objetivos institucionales y objetivos personales.

El clima laboral para la ejecución de proyectos, dicho por Villacrés y López (2018) debe desarrollar capacidades que colaboren para generar familiaridad en el equipo de gestión. Esto debe conllevar para tener auto confianza y auto conocerse a la interna, lo cual se logrará entender en base a las necesidades de los funcionarios. Esto debe encargarse para ser el precursor de un ambiente saludable, en la que los funcionarios se deban desarrollar en base al aprendizaje y en donde el error sirva únicamente para aprender y mejorar, de tal forma que los esfuerzos de la Gerencia se concentren en estos factores para garantizar los resultados; en este sentido para Román y Pérez (2018) el medio ambiente, el desarrollo humano y económico, componentes del clima laboral que propician una cultura que genere amabilidad, desarrollo, confort y satisfacción en el ámbito laboral. Con lo cual, es importante mencionar que el ambiente laboral se ha convertido en una situación compleja, la misma que no es ajena a la calidad de vida en lo referente a lo laboral, debido a que es influyente, directa o indirectamente, y contribuye al desarrollo institucional. En consecuencia, el ambiente laboral, su influencia sobre la conducta, satisfacción y en la productividad de los funcionarios se relaciona con el saber hacer (Rivera et al. , 2018). Asimismo, para contextualizarlo referido por Rivera et al. (2018) el clima labopral pùede derivar en diversas consecuencias físicas y psicológicas que producen desgaste para los colaboradores, provocando ausentismo, intención de ser removido, reubicación o estar inmersos en una rotación de personal, escasa productividad, rechazo laboral, inferioridad, insatisfacción y carencia de realización personal, desvinculaciones; en donde, la dirección y el liderazgo juegan un rol fundamental (Duran-Seguel, 2019), para favorecer la perdurabilidad institucional (Román y Pérez, 2018), en lo referente a proyectos, el clima laboral desarrolla la capacidad de armonizar intereses de los funcionarios y las direcciones con las que se relacionan e intervienen en ejecución de procesos (Duran-Seguel et al., 2019).

Ante lo expuesto, la presente investigación busca relacionar resultados de los proyectos BID en sus distintas etapas con la motivación dentro del ámbito laboral, permitiendo identificar cómo se activa, orienta, dinamiza y mantiene la conducta de los funcionarios hacia los objetivos esperados en la ejecución de los procesos, y con la satisfacción laboral como parte de una buena administración institucional. La investigación adicional busca dr luz a potenciales soluciones que enfrentan los proyectso BID, siendo necesario analizar la incidencia del clima laboral en la ejecución de los Contratos de Préstamo del Proyecto BID, a efecto de facilitar a la instancia correspondiente la información necesaria para la toma adecuada de decisiones encaminadas a alinear el comportamiento de los colaboradores a los objetivos institucioanles. La investigación permitirá identificar las características del clima laboral en la ejecución de los Contratos de Préstamo del Proyecto BID, para revisar cuáles son los componentes del clima laboral que se relacionan de manera positiva con los resultados que debe garantizarse en la ejecución de dichos proyectos, de tal forma que los esfuerzos de la Gerencia se concentren en estos factores que garantizaran los resultados que se persiguen.

\subsection{Clima laboral y sus dimensiones}

Serrano, P y Cruz, L (2008) mencionan que un óptimo clima laboral favorecerá la obtención: Mayor productividad, formación de líderes, identidad institucional, comunicación adecuada, reducción de conflictos, y que según García (2012) a creación de políticas es relevante en virtud de establecer un comportamiento en la institución y la forma como se deben hacer las cosas siendo guía para las actividades laborales de los funcionarios de la 
entidad, asumiendo y compartiendo responsabilidades de las actividades asignadas, esta particularidad brinda al equipo de gestión mesura y lo más relevante la confianza para tomar decisiones a beneficio del Proyecto y evitar percepciones negativas que alcancen a ser dañinas o perjudiciales para el equipo de gestión y el clima laboral, para lo cual Jiménez-Bonilla, (2016) argumenta que el clima laboral es muy importante y relevante dentro de una institución, en virtud que a los funcionarios les genera estabilidad para garantizar mayor productividad y beneficio para el Proyecto, creando un buen enlace en las comunicaciones ya que forman un papel preponderante en las relaciones interpersonales en el equipo de gestión.

De esta manera Chirinos, (2018) hace énfasis a la importancia de la interacción entre los funcionarios en las instituciones, debido al conocimiento sobre el ambiente en el cual se desarrollan para compensar sus necesidades esenciales, las cuales son: beneficios, ambiente de trabajo, mejora salarial, entre otros debemos destacar además que en toda institución se debe tener un sentido de reconocimiento, compartir valores, propósitos, oportunidades de enseñanza, aprendizaje y crecimiento, para poder fortalecer los procesos administrativos, financieros y de talento humano, que permita acrecentar la eficiencia y eficacia del Proyecto. Es importante mencionar la definición de Molano, (2018) en donde consideran factores que influyen en el clima laboral a las relaciones entre el equipo de gestión las cuales se refieren a la comunicación e información como de vital importancia, manejándose de manera respetuosa hacia todos los integrantes del Proyecto, siendo importante para obtener buenos resultados, el manejo del equipo por parte del líder o gerente del Proyecto.

Con lo cual, según Peña (2017), el clima laboral genera una gran ventaja competitiva, en donde compone mejores condiciones laborales que se reflejan en un mejor rendimiento administrativo, financiero, satisfacción de trabajar en equipo, manejo de una buena comunicación laboral, buen desenvolvimiento de cada uno de los funcionario que labora en la institución, logrando así la satisfacción laboral que para Arana et al. (2018) sintetiza sobre la información del ambiente laboral en acciones diarias en los diferentes departamentos, gerencias y direcciones para el logro de resultados. En ese sentido lo manifestado por D. Rivera, Rincón, et al., (2018) las dimensiones del clima laboral sobresalen atributos característicos como la libertad, autonomía, respaldo, justicia, creatividad, confianza, entre otros. Sin embargo, es esencial que al mismo tiempo exista un monitoreo por parte de la Dirección Nacional de Talento Humano ya que se debe contar con supervisión que avale el rendimiento del equipo, fundamentándose principalmente en las buenas relaciones personales y las experiencias de cada uno de los funcionarios dentro del proyecto. Conforme lo expuesto el presente estudio considera dimensión del clima laboral (Tabla 1) desde los siguientes enfoques:

Tabla 1

Dimensiones del Clima Laboral

\begin{tabular}{|c|c|l|}
\hline Dimensiones & Autores & \multicolumn{1}{c|}{ Enfoque } \\
\hline Autorrealización & $\begin{array}{c}\text { Maslow } \\
(1954)\end{array}$ & $\begin{array}{l}\text { Relaciona el término directamente con la madurez del individuo; también señala } \\
\text { como la manera en la que trasciende en sus necesidades básicas, permaneciendo } \\
\text { ligada a la realización personal y bienestar del entorno que lo rodea; además de } \\
\text { concluir que los sujetos poseen ciertas características en común como: Una } \\
\text { correcta percepción de la realidad, aprobación, espontaneidad, centrado de } \\
\text { problemas, privacidad, autonomía, evaluación clara, experiencia, sentido de } \\
\text { pertenencia con el equipo, sólidas relaciones a la vez que selectivas, estructura } \\
\text { democrática, convicción ética, humor, creatividad, resistencia a la adaptación, } \\
\text { valores, eliminación de dicotomías. }\end{array}$ \\
\hline Supervisión & Fayol y Taylor \\
$(1984)$, & $\begin{array}{l}\text { Establecen que la supervisión se basa en la valoración de los procesos alcanzados } \\
\text { en relación con los objetivos que persigue la empresa, busca principalmente } \\
\text { evidenciar las fortalezas y debilidades a fin de desarrollar un plan de acción que } \\
\text { genere nuevas actividades de mejoramiento; y que manifiesta detalladamente } \\
\text { como los mandos intermedios y dirección ayudaran con la interacción de los } \\
\text { colaboradores y toma de decisiones. }\end{array}$ \\
\hline
\end{tabular}




\begin{tabular}{|c|c|c|}
\hline Dimensiones & Autores & Enfoque \\
\hline $\begin{array}{c}\text { Involucramiento } \\
\text { Laboral }\end{array}$ & $\begin{array}{l}\text { Jaime et al., } \\
\text { (2016) }\end{array}$ & $\begin{array}{l}\text { Establecen que este aspecto es relacionado directamente con la presencia de } \\
\text { bases Institucionales fundamentadas sobre el recurso humano para ser partícipes } \\
\text { de las decisiones en las estructuras laborales, respondiendo de una manera } \\
\text { efectiva y enfocando los esfuerzos hacia la identificación institucional, se refieren } \\
\text { netamente a la percepción psicológica de como el colaborador se identifica con } \\
\text { los valores y objetivos Institucionales con el propósito principal que exista un } \\
\text { correcto trabajo en equipo y se desarrollen las actividades de manera eficaz; es } \\
\text { importante exponer que muchas veces el termino involucramiento laboral se } \\
\text { suele confundir con el compromiso laboral }\end{array}$ \\
\hline $\begin{array}{l}\text { Condiciones } \\
\text { Laborales }\end{array}$ & $\begin{array}{c}\text { Canales et al., } \\
(2016)\end{array}$ & $\begin{array}{l}\text { Se define a las condiciones laborales como: Cualquier característica que pueda } \\
\text { tener una influencia significativa en la generación de riesgos para la seguridad y } \\
\text { salud de los funcionarios, constituye un rol fundamental ya que se relacionan con } \\
\text { los recursos que la institución entrega a sus colaboradores, estos pueden ser: } \\
\text { insumos psicosociales o económicos para el desarrollo de las actividades que } \\
\text { ejecuten, además de estar enfocados principalmente hacia el bienestar y salud } \\
\text { del funcionario; cuando las condiciones laborales no son favorables generan } \\
\text { situaciones como: insatisfacción laboral, alteraciones físicas y psicológicas }\end{array}$ \\
\hline Comunicación & $\begin{array}{c}\text { Apolo,D } \\
\text { (2017) } \\
\text { Herrera, } \\
\text { (2018) }\end{array}$ & $\begin{array}{l}\text { Afirma que la comunicación es fundamental en la construcción de relaciones } \\
\text { entre instituciones, organismos y usuarios permitiendo establecer objetivos } \\
\text { colectivos y alcance de los mismos. } \\
\text { La comunicación nace a partir de un proceso donde se genera intercambio, } \\
\text { procesamiento, y almacenamiento de mensajes dentro de las instituciones, es } \\
\text { importante la colaboración colectiva ya que de esta manera se logran superar las } \\
\text { barreras y limitantes, además que la comunicación en la institución ayuda a: } \\
\text { realizar tareas relacionadas con las funciones asignadas, asimilar los cambios y } \\
\text { adaptación tanto individual como colectiva, apoyo en el cumplimiento de labores } \\
\text { en base a las políticas institucionales que desarrollan mecanismos para que la } \\
\text { información circule a las funcionarios dentro de la institución. Entrelazando estos } \\
\text { aspectos han permitido la evolución en el tiempo relacionando las características } \\
\text { coyunturales del entorno de las instituciones }\end{array}$ \\
\hline
\end{tabular}

\subsection{Proyectos BID y su ejecución}

Las característica deseables de los Proyectos según Sánchez et al., (2018) es la instauración del valor social. El mismo que podemos manifestar de varias formas, con las debidas precauciones concernientes al medio ambiente, beneficiando a la inclusión social, operando de forma eficaz y socialmente responsable, incidiendo sobre la comunidad que requieren de la atención social con calidad y calidez, la misma que no necesariamente es cubierta adecuadamente por el Estado, además de concretarse en las iniciativas de fórmulas que contribuyen a la creación de empleo estable, al desarrollo territorial y a la cohesión social. Los Proyectos según Paredes Gavilanez et al., (2019) tienen inicio y fin, los programas y procesos no son repetitivos, además de tener una estructura de organización transitoria y versátil, son ejecutables con de un presupuesto económico explícito y tienen solicitudes gerenciales a veces discordantes con la organización permanentemente.

Los Proyectos según Paredes Gavilanez et al., (2019) tienen inicio y fin, los programas y procesos no son repetitivos, además de tener una estructura de organización transitoria y versátil, son ejecutables con un presupuesto económico explícito y tienen solicitudes gerenciales a veces discordantes con la organización permanentemente. Los Proyectos BID tienen como objeto la mejora de la calidad en la prestación de los servicios sociales, consiste en fortalecer el diagnóstico temprano y la calificación de discapacidades desde la primera infancia, aumentar la disponibilidad de ayudas técnicas y mejorar el acompañamiento por parte de los servicios de salud y, contribuir a mejorar la calidad en la prestación de servicios sociales en Ecuador, particularmente en 
los sectores de educación y salud. Para la adjudicación de los procesos BID es de relevancia revisar toda la documentación que se generen dentro del Proyecto, previo a la suscripción de autorizaciones de inicio de proceso, resoluciones de inicio de proceso, resoluciones de adjudicación, contratos y demás documentos necesarios para su ejecución en la adquisiciones respectivas (bienes, obras y servicios, incluidos los de consultoría) (Tabla2).

Tabla 2

Fases, pasos y responsables en la ejecución de procesos del Proyecto BID

\begin{tabular}{|c|c|c|}
\hline Fases & Pasos & Responsables \\
\hline \multirow{5}{*}{$\begin{array}{l}\text { Preparatoria } \\
\text { Según Fernanda Mahecha Hernández y } \\
\text { Militar Nueva Granada, (2019) la fase } \\
\text { preparatoria, consiste en identificar una } \\
\text { serie de necesidades prioritarias de } \\
\text { acuerdo con la misionalidad de la } \\
\text { institución para la contribución del logro } \\
\text { de los fines del Estado por medio del } \\
\text { diseño del proyecto. }\end{array}$} & $\begin{array}{l}\text { Elabora justificación de la necesidad, estudio } \\
\text { de mercado, Términos de Referencia, } \\
\text { prepara expediente (certificación PA, PAPP, } \\
\text { Aval, certificación presupuestaria) } \\
\text { Elaborar Documento de Licitación. }\end{array}$ & Áreas requirentes (Direcciones) \\
\hline & Solicitar No Objeción al BID. & Equipo de Gestión (EDG) Proyecto BID \\
\hline & Banco emite No Objeción. & Banco Interamericano de Desarrollo \\
\hline & Conformar Comisión Técnica. & EDG Proyecto BID. \\
\hline & $\begin{array}{l}\text { Solicitar elaboración de Resolución de Inicio. } \\
\text { Revisar y Elaborar Resolución de Inicio. }\end{array}$ & $\begin{array}{l}\text { Dirección Nacional de Contratación } \\
\text { Pública }\end{array}$ \\
\hline \multirow[t]{5}{*}{$\begin{array}{l}\text { Precontractual } \\
\text { La fase precontractual es la que realiza el } \\
\text { pliego, misma que se encarga de } \\
\text { estipular cláusulas y parámetros para } \\
\text { adjudicación, a la vez ejecutar un } \\
\text { contrato, según Fernanda Mahecha } \\
\text { Hernández y Militar Nueva Granada, } \\
\text { (2019) se delega a un grupo de expertos } \\
\text { (comité técnico) para la formulación del } \\
\text { documento en mención para aplicar } \\
\text { condiciones. }\end{array}$} & $\begin{array}{l}\text { Publicar el llamado a licitación (UNDB/Portal } \\
\text { electrónico/periódico nacional). } \\
\text { Solicitud de aclaraciones sobre el documento } \\
\text { de licitación por participantes. } \\
\text { Preparación de respuestas sobre solicitudes } \\
\text { de aclaración y envío a los participantes. } \\
\text { Preparación de enmienda (s) del documento } \\
\text { de licitación. } \\
\text { Solicitar No objeción del Banco a la (s) } \\
\text { enmienda (s). }\end{array}$ & EDG Proyecto BID \\
\hline & BID emite No Objeción. & Banco Interamericano de Desarrollo. \\
\hline & $\begin{array}{l}\text { Preparación y evaluación de ofertas. } \\
\text { Solicitar No objeción al Banco al informe de } \\
\text { evaluación de ofertas. }\end{array}$ & EDG Proyecto BID \\
\hline & BID emite No Objeción. & Banco Interamericano de Desarrollo. \\
\hline & Adjudicación y publicación del contrato. & EDG Proyecto BID \\
\hline \multirow{3}{*}{$\begin{array}{l}\text { Contractual } \\
\text { Es la ejecución contrato suscrito, } \\
\text { conforme el cumplimiento del } \\
\text { cronograma y plazos establecidos; y } \\
\text { Fernanda Mahecha Hernández y Militar } \\
\text { Nueva Granada, (2019) es el proceso en } \\
\text { el cual se legaliza el contrato. }\end{array}$} & $\begin{array}{l}\text { Elaboración de contrato. } \\
\text { Protocolización. Domiciliación. } \\
\text { Entrega de Garantías. }\end{array}$ & $\begin{array}{c}\text { Coordinación General de Asesoría } \\
\text { Jurídica }\end{array}$ \\
\hline & $\begin{array}{l}\text { Suscripción del contrato (garantías, } \\
\text { protocolización del documento). }\end{array}$ & $\begin{array}{c}\text { Gerencia del Proyecto BID/ Proveedor } \\
\text { Adjudicado. }\end{array}$ \\
\hline & $\begin{array}{l}\text { Solicitar Código Prism } \\
\text { Genera código Prism }\end{array}$ & EDG Proyecto BID \\
\hline \multirow{2}{*}{$\begin{array}{l}\text { Poscontractual } \\
\text { Elabora y Suscribe según Fernanda } \\
\text { Mahecha Hernández y Militar Nueva } \\
\text { Granada, (2019) las actas de recepción } \\
\text { temporal, recepción definitiva y actas de } \\
\text { liquidación; previo a la terminación de } \\
\text { ejecución del contrato. }\end{array}$} & $\begin{array}{l}\text { Entrega del Primer producto (actas). } \\
\text { Entrega del Segundo producto (actas). } \\
\text { Entrega del Tercer producto (actas). } \\
\text { Revisión y aprobación del producto. }\end{array}$ & $\begin{array}{l}\text { EDG Proyecto BID/ Dirección (área } \\
\text { requirente) / Administrador de } \\
\text { contrato. }\end{array}$ \\
\hline & Ejecución del pago. & Dirección Nacional Financiera \\
\hline
\end{tabular}

Elaborado: por los autores

Según Lejarriaga Pérez de las Vacas et al., (2018) las características de un Proyecto se encamina a la percepción del emprendimiento social, en donde, la característica deseable para Sánchez Espada et al., (2018) es la 
instauración del valor social, que puede manifestarse de varias formas con las debidas precauciones concernientes al medio ambiente, beneficiando a la inclusión social, operando de forma eficaz y socialmente responsable, incidiendo sobre la comunidad que requieren de la atención social con calidad y calidez, la misma que no necesariamente es cubierta adecuadamente por el Estado, es así que además de concretarse en las iniciativas de fórmulas contribuyen a la creación de empleo estable, al desarrollo territorial y a la cohesión social.

\section{Metodología}

\subsection{Modalidad de investigación}

Para evaluar la eficiencia de un Proyecto, se consideró en la revisión teórica los avances de las variables motivo de estudio de manera cronológica a fin de analizar su nivel o grado de relación, logrando identificar las dimensiones del clima laboral que deben ser analizadas en el estudio, relacionadas a Autorrealización, Involucramiento Laboral, Supervisión, Comunicación, Condiciones laborales, así como, se definió para la variable proyectos la adjudicación de procesos y las direcciones que intervienen en el mismo, considerando las fases preparatoria, precontractual, contractual y postcontractual con las que cuenta el Proyecto BID, permitiendo contar con las bases teóricas que orientaran el presente estudio en el análisis de la forma en la que se relacionar y garantizan el éxito de un proyecto. Luego, el clima laboral, abarca la interacción entre todos los componentes de la institución, que generan un ambiente, y las características psicológicas de los funcionarios. Con el fin de conocer cual es el grado de satisfacción del clima laboral y su incidencia con el comportamiento de los funcionarios dentro de las áreas inmersas en la ejecución de los préstamos BID se empleó un análisis correlacional de acuerdo con A. Rivera et al., (2018) este estudio se realiza bajo la metodología de investigación cuantitativa, debido a que se recolectaran y analizaran datos de las variables de estudio de clima laboral con la dimensión variable de proyectos en los funcionarios del Proyecto BID.

\subsection{Población y muestra}

La población para la ejecución de los procesos BID son de 38 funcionarios, con lo cual, se considera dentro de la muestra de estudio a la totalidad de la población, siendo una técnica recomendada por Godoy, (2019) para poblaciones de estas características. Este estudio se procedió a ingresar la encuesta en la herramienta SuverMonckey para remitirla a través de la aplicación WhatsApp a los diferentes números telefónicos de los funcionarios que intervienen en la ejecución de procesos BID, lo cual se solicitó el apoyo de la asistente del Proyecto con un listado facilitado de la parte investigativa, para que haga la indagación respectiva con la finalidad de conseguir cada uno de los contactos de los funcionarios; posterior a ello, se envió a cada contacto el link de la encuesta, considerando que no se pudo aplicar de manera física por las condiciones de aislamiento dadas por el Gobierno ante la emergencia sanitaria debido a la pandemia del COVID-19.

\subsection{Instrumento}

La investigación emplea como instrumento de estudio la encuesta que es una forma recomendado por Alvarado et al., (2013) para obtener información verás que pueda ser contrastada estadísticamente, mediante aplicación de preguntas que estén dirigidas a los funcionarios que intervienen en los Proyectos BID, empleando para medir las dimensiones del clima laboral de autorrealización, involucramiento laboral, supervisión, comunicación, condiciones laborales, el modelo que emplea la herramienta de medición CL-SPC desarrollada por Palma (2004); en el caso de la variable de Proyectos se emplea el cuestionario recomendado por Trujillo, (2017) que está bajo una licencia de Creative Commons Reconocimiento-NoComercial-Compartirlgual 3.0. 


\subsection{Levantamiento de información}

Para determinar la relación del clima laboral en los colaboradores del proyecto, el levantamiento de información fue apoyado en base a la escala CL-SPC que toma en cuenta ámbitos vitales para el presente estudio. De esta manera, en un universo de 38 funcionarios involucrados de los cuales 22 son de género masculino y 16 femenino lograr medir la percepción de cada uno de los ámbitos para determinar los puntos críticos que inciden en el clima laboral ( Bravo y Calderón ,2017; Rivera, 2018; Jiménez y Mariño, 2018). Para el levantamiento de la información la encuesta se basa en la escala de Likert que involucra 5 opciones: siempre, mucho, a veces, poco y nunca. Partiendo de una percepción favorable que va disminuyendo según van pasando las alternativas hasta llegar al punto mínimo de "nunca" que expresa total desconformidad con la afirmación de la encuesta. En cada dimensión se puede observar una tabla que indica la frecuencia con la cual los funcionarios escogieron cada una de las opciones, dependiendo de las preguntas, y su frecuencia relativa.

\section{Resultados}

Recabada la información en base a las encuestas enviadas de cada funcionario, se procedió a cargar la base de datos a la herramienta SPSS; constatando el número de encuestas válidas para el análisis descriptivo en primera instancia y en lo posterior el análisis correlacional en cumplimiento de los objetivos que guiaron la presente investigación. En relación a los resultados obtenidos para dar respuesta al objetivo de Identificar las características del clima laboral en la ejecución de los Contratos de Préstamo del Proyecto BID y lograr determinar los factores dominantes, de acuerdo con lo mencionado por Román y Pérez (2018) el levantamiento de la información fue realizado a través de encuestas basadas en diversas escalas de repuestas estandarizadas, que hacen posible establecer tendencias en las diferentes mediciones, tomando en cuenta aspectos que inciden directamente con la percepción de cada funcionario de cómo se manifiesta el ambiente de trabajo. Para el clima laboral se encarga de mostrar las diversas dimensiones y así conocer los resultados de manera general de cada una de las variables, en las que se indica la percepción global de los funcionarios en las encuestas realizadas ver (tabla 3). Mamani, (2018) explica que el clima laboral está estrechamente vinculado con el nivel de satisfacción de los colaboradores, manifestando que cuando la percepción es alta mejoran las relaciones de satisfacción, animo, interés, colaboración, cooperación, etc. Por lo contrario, si el clima laboral tiene tendencias negativas en el grupo de trabajo, se puede manifestar desinterés, apatía, depresión, conflictos internos, inconformidad, perjudicando notablemente el alcance de los objetivos institucionales.

En cuanto a la correlación entre el clima laboral con el logro de resultados en la ejecución de los Contratos de Préstamo del Proyecto BID, dado que en este estudio se ha enfocado en identificar si existe relación entre las variables clima laboral y la ejecución de los Contratos de Préstamo BID. Se consideró la correlación de Pearson, tomando en cuenta que las variables analizadas son categóricas y que la muestra fue seleccionada al azar. Existe igualdad en la distribución de la muestra a nivel bilateral por lo cual es viable el uso de Correlación de Pearson.

Se realizaron dos tablas para consolidar las variables, tomando en cuenta sus dimensiones, las que se encuentran catalogadas en la Escala de Likert, en el caso del Clima Laboral y fases en el caso de ejecución de los Contratos. Para reconocer los niveles de cada fase y dimensión, se realizó una sumatoria de las dimensiones de las variables para obtener un total de las variables, se caracterizó esta sumatoria en función de los niveles, convirtiéndolas en variables categóricas.

\section{Hipótesis:}

Ho El clima laboral no tiene relación con la ejecución de los Contratos de Préstamo del Proyecto BID H1 El clima laboral incide en la ejecución de los Contratos de Préstamo del Proyecto BID. 
En la escala de los rangos de coeficiente de correlación de Pearson se consideró una escala de entre -1 y 1 ; por lo que se interpretaron de la siguiente manera según se menciona en la (tabla 5).

Tabla 3

Escala de Likert Clima Laboral

\begin{tabular}{cccccc}
\hline & \multicolumn{5}{c}{ Frecuencias totales por dimensión } \\
& Nunca & Poco & A veces & Mucho & Siempre \\
\hline Autorrealización & 36 & 69 & 92 & 41 & 28 \\
\hline Involucramiento & 18 & 60 & 93 & 80 & 53 \\
\hline Supervisión & 37 & 111 & 105 & 62 & 27 \\
\hline Comunicación & 31 & 141 & 203 & 110 & 47 \\
\hline Condiciones laborales & 50 & 92 & 135 & 70 & 33 \\
\hline TOTAL & 172 & 473 & 628 & 363 & 188 \\
\hline Porcentaje & $9.43 \%$ & $25.93 \%$ & $34.43 \%$ & $19.90 \%$ & $10.31 \%$ \\
\hline & Fuente y elaboración: autores & & \\
\hline
\end{tabular}

Tabla 4

Escala de Likert Fases de Ejecución.

\begin{tabular}{cccccc}
\hline & \multicolumn{5}{c}{ Frecuencias totales por dimensión } \\
\hline & Nunca & Poco & A veces & Mucho & Siempre \\
\hline Preparatoria & 30 & 86 & 95 & 41 & 14 \\
\hline Precontractual & 24 & 83 & 99 & 37 & 23 \\
\hline Contractual & 23 & 40 & 51 & 24 & 14 \\
\hline Poscontractual & 58 & 123 & 149 & 74 & 52 \\
\hline TOTAL & 135 & 332 & 394 & 176 & 103 \\
\hline PORCENTAJE & $11.84 \%$ & $29.12 \%$ & $34.56 \%$ & $15.44 \%$ & $9.04 \%$ \\
\hline
\end{tabular}

Fuente y elaboración: autores

Tabla 5

Rangos de Coeficiente de Correlación de Pearson

\begin{tabular}{|c|c|c|}
\hline Coeficiente de la correlación & Significado & Interpretación \\
\hline "-1 & Correlación negativa grande y perfecta & \multirow{6}{*}{$\begin{array}{l}\text { Las variables de relacionan } \\
\text { inversamente }\end{array}$} \\
\hline "-0.9 a -0.99 & Correlación negativa muy alta & \\
\hline "-0.7 a -0.89 & Correlación negativa alta & \\
\hline "-0.4 a -0.69 & Correlación negativa moderada & \\
\hline "-0.2 a -0.39 & Correlación negativa baja & \\
\hline "-0.01 a -0.19 & Correlación negativa muy baja & \\
\hline "0 & Correlación nula & Las variables son independientes \\
\hline "0.19 a 0.19 & Correlación positiva muy baja & \multirow{6}{*}{$\begin{array}{l}\text { Las variables se correlacionan } \\
\text { directamente }\end{array}$} \\
\hline "0.2 a 0.39 & Correlación positiva baja & \\
\hline "0.4 a 0.69 & Correlación positiva moderada & \\
\hline "0.7 a 0.89 & Correlación positiva alta & \\
\hline "0.9 a 0.99 & Correlación positiva muy alta & \\
\hline "1 & Correlación positiva grande y perfecta & \\
\hline
\end{tabular}

Elaboración: autores 
Realizado el análisis correlacional entre las dimensiones del clima laboral y las fases del proyecto BID se obtuvieron los siguientes resultados que se refieren en la (tabla 6):

Tabla 6

Correlación de Pearson Proyectos

\begin{tabular}{|c|c|c|c|c|c|c|c|c|c|}
\hline \multirow[b]{3}{*}{ Clima laboral } & \multicolumn{8}{|c|}{ Proyectos } & \multirow[b]{3}{*}{$\begin{array}{c}\text { TOTAL, } \\
\text { CORRELACIÓN }\end{array}$} \\
\hline & \multicolumn{2}{|c|}{ Preparatoria } & \multicolumn{2}{|c|}{ Precontractual } & \multicolumn{2}{|c|}{ Contractual } & \multicolumn{2}{|c|}{ Poscontractual } & \\
\hline & $\begin{array}{c}\text { Coeficiente } \\
\text { de } \\
\text { correlación }\end{array}$ & $\begin{array}{l}\text { Coeficiente de } \\
\text { determinación }\end{array}$ & $\begin{array}{c}\text { Coeficiente } \\
\text { de } \\
\text { correlación }\end{array}$ & $\begin{array}{l}\text { Coeficiente de } \\
\text { determinación }\end{array}$ & $\begin{array}{c}\text { Coeficiente } \\
\text { de } \\
\text { correlación }\end{array}$ & $\begin{array}{l}\text { Coeficiente de } \\
\text { determinación }\end{array}$ & $\begin{array}{c}\text { Coeficiente } \\
\text { de } \\
\text { correlación }\end{array}$ & $\begin{array}{l}\text { Coeficiente de } \\
\text { determinación }\end{array}$ & \\
\hline \multicolumn{10}{|l|}{ Autorrealizació } \\
\hline $\mathrm{n}$ & 0.791 & $62.57 \%$ & 0.686 & $47.06 \%$ & 0.75 & $56.25 \%$ & 0.795 & $63.20 \%$ & 3.022 \\
\hline \multicolumn{10}{|l|}{ Involucramient } \\
\hline 0 & 0.567 & $32.15 \%$ & 0.44 & $19.36 \%$ & 0.546 & $29.81 \%$ & 0.572 & $32.72 \%$ & 2.125 \\
\hline Supervisión & 0.828 & $68.56 \%$ & 0.694 & $48.16 \%$ & 0.761 & $57.91 \%$ & 0.845 & $71.40 \%$ & 3.128 \\
\hline Comunicación & 0.817 & $66.75 \%$ & 0.701 & $49.14 \%$ & 0.776 & $60.22 \%$ & 0.828 & $68.56 \%$ & 3.122 \\
\hline \multicolumn{10}{|l|}{ Condiciones } \\
\hline laborales & 0.826 & $68.23 \%$ & 0.679 & $46.10 \%$ & 0.736 & $54.17 \%$ & 0.829 & $68.72 \%$ & 3.07 \\
\hline TOTAL & 3.829 & 2.982519 & 3.2 & 2.098274 & 3.569 & 2.583609 & 3.869 & 3.046059 & \\
\hline
\end{tabular}

Elaboración: autores

Tabla 7

Coeficientes

\begin{tabular}{ccc}
\hline & $\begin{array}{c}\text { Coeficiente de } \\
\text { correlación }\end{array}$ & $\begin{array}{c}\text { Coeficiente de } \\
\text { determinación }\end{array}$ \\
\hline Máximo & 0.845 & $71.40 \%$ \\
\hline Mínimo & 0.44 & $19 \%$ \\
\hline Media & 0.7153125 & $54 \%$ \\
\hline
\end{tabular}

Fuente y elaboración: autores

Se puede observar que existe relación fuerte entre ambas variables, por lo que se rechaza la hipótesis nula y se acepta H1. Es así como podemos observar en la tabla 7 el coeficiente de correlación de cada dimensión se encuentra en un rango entre 0,44 y 0,84 ; es decir, que tiene una correlación positiva entre alta y moderada como se muestra en la tabla 6, y que todas las dimensiones del clima laboral tienen influencia en la ejecución de las fases de los contratos, siendo la dimensión con mayor influencia la Supervisión frente a la etapa Poscontractual en donde se da a notar un solido coeficiente de correlación con un valor de 0.84 entre ambas variables; revelando el estado emocional y de seguridad que tienen los funcionarios de la Institución, por este motivo Jaime et al., (2016) establecen que se relaciona directamente con la presencia de bases Institucionales fundamentadas sobre el recurso humano para ser partícipes de las decisiones en las estructuras laborales, respondiendo de una manera efectiva y enfocando los esfuerzos hacia la identificación institucional; tambien se debe tomar en cuenta que Jaime et al., (2016) plantean que este aspecto es relacionado directamente con la presencia de bases Institucionales fundamentadas sobre el recurso humano para ser partícipes de las decisiones en las estructuras laborales, respondiendo de una manera efectiva y enfocando los esfuerzos hacia la identificación institucional.

A continuación se puede notar que existe una fuerte influencia entre las variables de supervisión frente a la fase preparatoria y la supervisión frente a la etapa poscontractual pues ambas comparten un valor fuerte en cuanto al coeficiente de correlacion, indicando un valor de 0.82 en ambas correlaciones donde por parte de la supervisión frente a la fase preparatoria se puede notar que abarca información rutinaria sobre diferentes aspectos en el proyecto, a fin de dar seguimiento basado en apreciación y funcionalidad por parte de los lideres 
del equipo a la vez que se busca invitar a alternativas de evolución en el ámbito laboral y económico, a finde que mejoren el desempeño y calidad en el trabajo en el que se desenvuelven; por otro lado la supervisión frente a la etapa poscontractual apuntan los esfuerzos hacia mejoramiento continuo o capacitación Arias, (2019) manifiesta que las instituciones que cuentan con un plan de carrera obtienen ventajas en el desarrollo de los oficinistas con potencial y memoria institucional, una de las cuestiones cuenta en la disminución de la tasa de rotación de personal.

Al analizar los resultados de los niveles mas bajos de correlación entre las variables, se situan en una correlación positiva moderada; en cuanto al involucramiento versus la etapa precontractual, se indica que el valor mas bajo es de 0.44 en el coeficiente de correlación, lo cual se refiere a estipular cláusulas y parámetros para adjudicación al ejecutar un contrato; de acuerdo con lo mencionado por Zepeda et al., (2016), al existir óptimas condiciones en el desarrollo de este ámbito el mismo incita a la mejora de la calidad de trabajo, productos, trabajo en equipo y mejora las destrezas competitivas en las instituciones u organizaciones.

Se puede notar que la correlación con mas bajo nivel entre sus variables es el involucramiento frente a la etapa contractual indicando un valor de 0.54 de coeficiente, donde es importante verificar si los funcionarios se sienten identificados con los valores y objetivos institucionales, según lo planteado por Tarillo (2019); se evaluó que las actividades deben realizarse conforme el cumplimiento del cronograma y plazos establecidos, así como el cumplimiento de las directrices y clausulas determinadas. Finalmente el involucramiento frente a la etapa preparatoria revela que es la tercera etapa mas critica con 0.56 de coeficiente de correlación donde, los indicadores muestran que las actividades no se desarrollan siguiendo métodos o planes establecidos, a la vez que el equipo de gestión del proyecto no representan un soporte para el resto de funcionarios siendo de importancia considerar los métodos de liderazgo, comunicación, toma de decisiones, control, relaciones interpersonales, motivación, capacitación y entrenamiento que sería relevante para los objetivos de la institución (Arce, 2017). Siendo así, para satisfacer a los funcionarios el coeficiente de determinación se define en la proporción de varianza compartida entre ambas variables, y se puede interpretar en que porcentaje de la ejecución de Proyectos depende de cada dimensión del clima laboral.

\section{Conclusiones}

Dentro del análisis cuantitativo de la percepción del clima laboral se puede concluir que existen varios ámbitos sobre los cuales se deben tomar medidas; en cuanto a la autorrealización, los resultados resaltan la falta de oportunidades de progresar en la Institución orientándose a la variable de acuerdo lo mencionado por Mart et al. (2020) estas condiciones originan consecuencias perjudiciales en el desarrollo del Proyecto, así como el deterioro progresivo del clima laboral.

En cuanto al involucramiento laboral, los indicadores muestran que las actividades no se desarrollan siguiendo métodos o planes establecidos, a la vez que el equipo de gestión del proyecto no representan un soporte para el resto de funcionarios; por este motivo, el indicador muestra una tendencia negativa en ambos aspectos de acuerdo con lo planteado por Cuya y Hiyane (2019) en el caso de corregir los inconvenientes y mantener este ámbito positivo los funcionarios se identificaran psicológicamente con su empleo y consideraran su desempeño como beneficio personal aumentando su rendimiento.

El ámbito de la comunicación se revela un enfoque negativo refiriéndose que la interacción con los de Nivel Jerárquico Superior es dificultosa, al mismo tiempo que la Institución no promueve y alienta la comunicación entre sus miembros, por lo que podrían suscitarse inconvenientes entre funcionarios, aumentando los malos entendidos, presencia de conflictos y en últimas instancias la destitución, lo que conlleva al desempleo de los funcionarios (Zarsoza y Barzola, 2019). La medición de las condiciones laborales revela que la Institución es una mala alternativa para alcanzar estabilidad laboral, de acuerdo con lo planteado por Castillo y Salas (2018) al 
existir inestabilidad, los funcionarios son más propensos a dejar sus puestos de trabajo y con el tiempo puede repercutir su salud, a la par disminuye la satisfacción con el trabajo. Las condiciones laborales revelan que la Institución no provee de los recursos tecnológicos necesarios para facilitar el trabajo, dificultando la mejora y desempeño del personal, además de tener la percepción que el salario no va acorde con el desempeño y los logros alcanzados; condiciones que contribuyen a una mayor incidencia de accidentes ocupacionales $y$ enfermedades, menor desempeño, estrés, fatiga e inconformidad con el trabajo (Reyes Ortiz, 2016).

La investigación evidencia como elementos fundamentales que la ejecución de los Contratos de Préstamo del BID deben garantizar la designación de funciones en la mayoría de los funcionarios muestran inseguridad con respecto a la asignación los mejores perfiles para las designaciones de los Comités Técnicos Evaluadores para los procesos de adjudicación de obras, bienes y servicios; asimismo los funcionarios tienen inconformidad respecto a la prontitud en la entrega de información que valida el manejo del proceso con la finalidad de cumplir con las etapas para la ejecución; en donde los funcionarios manifiestan que no existe privacidad en los procesos, pese haber suscrito un Acuerdo de Confidencialidad, siendo este un instrumento fundamental para evitar filtrar datos e información de los oferentes participantes, en virtud de aquello, es esencial el sigilo de información para tomar decisiones acordes que permitan emitir informes de recomendación de adjudicación por parte del Comité Técnico Evaluador.

El estudio reconoce como una posible limitación las condiciones laborales como efecto de la pandemia generada por el COVID 19, situación que puede generar en los encuestados sentimiento de intranquilidad por las desvinculaciones en el sector público, así como de la rotación del Nivel Jerárquico Superior dentro de las Direcciones y Gerencias en la Institución lo cual hace que los funcionarios tengan una elevada intranquilidad por el manejo de sus actividades y competencias. Los resultados obtenidos en respuesta a los objetivos específicos, permitirá a los profesionales en el campo de la administración tener una visión clara del comportamiento de las variables analizadas. Es así, en cuanto a la correlación del clima laboral con el logro de resultados en la ejecución de los Contratos de Préstamo del Proyecto BID, según el marco teórico, generan compromiso en los funcionarios donde se detallaron dentro de las dimensiones del clima laboral la Autorrealización, Involucramiento Laboral, Supervisión, Comunicación y Condiciones Laborales, en los resultados se detectaron relevantes conclusiones conjuntamente con las características del Proyecto, en donde se enmarca y se recalca la adjudicación de los procesos BID y sus etapas preparatoria, precontractual, contractual, poscontractual.

\section{Referencias bibliográficas}

Alvarado, A., Hurtado, B., \& Yávar, K. (2013). Universidad De Guayaquil Escuela De Ingeniería COMERCIAL Tesis que se presenta como requisito previo para la obtención del Título de Ingeniero Comercial en la Especialización de Recursos Humanos TUTOR :

Apolo, D., Baez, V., Pauker, L., \& Pasquel, G. (2017). Gestión de Comunicación Corporativa: consideraciones para el abordaje de su estudio y práctica. 521-539. https://doi.org/10.4185/RLCS

Arana, Carlos y Loyola, I. (2018). Facultad De Negocios.

Arce, R. (2017). Relación del clima organizacional con el desempeño de los comisarios , comisarías tipo " A " de la VII DIRTEPOL - año 2012. 183.

Arias, S. (2019). Identificación De Las Prácticas De Talento Humano Que Aumentan El Compromiso Laboral De Una Empresa Tecnológica En La Ciudad Quito. In Methods.

Bravo, M., \& Calderón, O. (2017). El Clima Laboral y su incidencia en el Desempeño del Personal Docente de una Escuela de Educación Básica en Ecuador. Podium, 1, 131-143. 
Canales, M., Valenzuela, S., \& Paravic, T. (2016). Condiciones de trabajo de los profesionales de enfermería en Chile. Enfermería Universitaria, 13(3), 178-185.

Castillo, J., \& Salas, C. (2018). Estabilidad Laboral Y Desigualdad Del Ingreso: Una Perspectiva De Género. Cuestiones Económicas, 28, 149-180.

Chirinos, Y. (2018). EL Clima Organizacional en emprendimiento sostenible. https://doi.org/10.21158/01208160.n84.2018.1916

Cuya, E., \& Hiyane, Y. (2019). Carrera de Administración de Empresas Involucramiento Organizacional Y Satisfacción Laboral En Una Institución Estatal , Lima 2019 Asesor : Ernesto Ángel Gustavo Manfredi Gagliuffi.

Duran-Seguel, I., Gallegos, M., \& Cabezas, D. (2019). Estilos de liderazgo y su influencia en el clima laboral: caso de estudio de una empresa exportadora de alimentos Leadership styles and their influence on working environment: case study of a food export company Contenido. In ISSN (Vol. 40).

Fayol, H., \& Taylor, F. (1984). Administracion general y industrial (p. 149).

Fernanda Mahecha Hernández, D., \& Militar Nueva Granada, U. (2019). I Los procesos contractuales de las Asociaciones Público-Privadas colombianas: Cuarta Generación de Concesiones Viales.

García, M. (2012). clima-laboral-empresa-competitividad.

Godoy, J. (2019). Percepción De Las Beneficiarias Del Programa Social Juntos En El Distrito De Capachica, 2017.

Herrera, C. (2018). Modelo estratégico de comunicación organizacional para la Facultad de Especialidades Empresariales de la Universidad Católica de Santiago de Guayaquil. Universidad Católica de Santiago de Guayaquil.

Jaime, J., Rodríguez, Z., Zulema, Z., Peraza, D., Karas, J. S., Paul, J., \& Manzanares, S. (2016). Investigación Involucramiento Laboral Y Productividad En Un Agronegocio En Sinaloa, Mexico. 19, 71-80.

Jiménez-Bonilla, D. (2016). Vista de Clima laboral y su incidencia en la satisfacción de los trabajadores de una empresa de consumo masivo. http://ojs.unemi.edu.ec/index.php/cienciaunemi/article/view/301/263

Jiménez, F., \& Mariño, I. (2018). Investigación y análisis de la rotación del talento humano en las cadenas de comidas rápidas y su impacto en la productividad y el clima laboral. Dominio de Las Ciencias, 4(3), 449. https://doi.org/10.23857/dc.v4i3.818

Lejarriaga Pérez de las Vacas, G., Bel Durán, P., Martín López, S., \& Sánchez Espada, J. (2018). Educación y formación en emprendimiento social: características y creación de valor social sostenible en proyectos de emprendimiento social. REVESCO. Revista de Estudios Cooperativos, 0(0), 16-38.

https://doi.org/10.5209/reve.62492

Mamani, L. (2018). Estrés Y Clima Laboral En El Personal Administrativo De La Dirección Regional Agraria Puno, 2018. Tesis, 1-13.

Mart, N., Villalobos, J., \& Machado, M. (2020). Autorrealización : elaboración de la escala ADO-20 para su evaluación en docentes. Self-realization : preparation of the ADO-20 scale for its assessment in Introducción En la sociedad actual , que está entre la moda, las exigencias sociales, el en su d. 3, 181-211.

Maslow, A. (1954). Motivation and personality. New York: Harpers.

Molano, A. (2018). Universidad Militar Nueva Granada Facultad De Ciencias.

Palma, S. (2004). Escala Clima Laboral CL - SPC Manual (1 Edición). Manual SPC 1 Edición, 52. 
Paredes Gavilanez, J. G., Cuvi, C., Raúl, V., Castelo Salazar, Á. G., \& Rojas Jara, H. V. (2019). Formulación de proyectos de inversión para las Pymes. Observatorio de La Economía Latinoamericana, enero.

Peña, J. (2017). Unidad Académica De Ciencias Empresariales.

Reyes Ortiz, A. (2016). Condiciones laborales y la satisfacción laboral de los colaboradores de la Comisión Nacional para el Desarrollo y Vida sin Drogas - Oficina Zonal Tarapoto, año 2016. Universidad César Vallejo.

Rivera, A., Hernández, D., Forgiony, J., Bonilla, N., \& Rozo Astrid. (2018). Impacto de la motivación laboral en el clima organizacional y las relaciones interpersonales en los funcionarios del sector salud. 39(16).

Rivera, D., Hernández, J., \& Forgiony, J. (2018). Impacto de la motivación laboral en el clima organizacional y las relaciones interpersonales en los funcionarios del sector salud (Vol. 39, Issue 16).

Rivera, D., Rincón, J., \& Flores, S. (2018). Percepción del Clima organizacional: Un análisis desde los Macroprocesos de una ESE. http://bonga.unisimon.edu.co/handle/20.500.12442/2166

Robbins, S. P., Enríquez Brito, J., Judge, T. A., María García, E., Manuel Juárez, J. H., Murillo, E., \& Leticia Ochoa Ramos Rita Guadalupe Franco Rosalinda Garza Estrada José Badajoz Gutiérrez, A. (2009). Decimotercera edición.

Robles, Dierssen, Martínez, Herrera, Díaz, \& Llorca, 2005 - Buscar con Google. (n.d.). Retrieved March 2, 2020, from

https://www.google.com/search?rlz=1C5CHFA_enEC698EC698\&sxsrf=ALeKk00mQddcVxLtzIS8Ypn_pnWY POSTdw\%3A1583202373465\&ei=RcBdXq7uG4-

Y_QbKs76YBg\&q=Robles\%2C+Dierssen\%2C+Martínez\%2C+Herrera\%2C+Díaz\%2C+\%26+Llorca\%2C+2005\& oq=Robles\%2C+Dierssen\%2C+Martínez\%2C+Herrera\%2C+Díaz\%2C+\%26+Llorca\%2C+2005\&gs_l=psyab.3...873662.873662..878578...0.0..0.255.399.0j1j1.....0....2j1..gws-

wiz.pFMXTZkQKsI\&ved=0ahUKEwjupM-4oP3nAhUPTN8KHcqZD2MQ4dUDCAs\&uact=5

Román, E., \& Pérez, E. (2018). Impacto de la responsabilidad social empresarial en el clima organizacional (Vol. 39).

Sánchez Espada, J., Martín López, S., \& Bel Durán Gustavo Lejarriaga, P. (2018). Educación y formación en emprendimiento social: características y creación de valor social sostenible en proyectos de emprendimiento social. https://doi.org/10.5209/REVE.62492

Serrano, p y Cruz, L. (2008). Estudio sobre clima y satisfacción laboral en una empresa comercializadora.

Tarillo, L. (2019). Teorías y modelos: formas de representación de la realidad. Comunicación, 12(1), 33-46. https://doi.org/10.18845/rc.v12i1.1212

Trujillo, F. (2017). Cuestionarios Proyectos.

Villacrés, J., \& López, H. (2018). Incidencia del tipo de liderazgo en el clima laboral.

Zarsoza, G., \& Barzola, R. (2019). Universidad De Guayaquil Facultad De Comunicación Social Carrera De Comunicación Social Título De Licenciado En Comunicación Social Del less Del Cantón Durán Para El Correcto Ing . Maria De Lourdes Zambrano Calvache . 2 Repositorio Nacional En Ciencia Y T.

Zepeda, J., Delgado, Z., Peraza, J., \& Soto, J. (2016). Investigación Involucramiento Laboral Y Productividad En Un Agronegocio En Sinaloa, Mexico. 19, 71-80.

Esta obra está bajo una Licencia Creative Commons Attribución-NoCommercial 4.0 International

(c) EY-NC 